\title{
A Passive Refillable Intraocular MEMS Drug Delivery Device
}

\author{
Ronalee $\mathrm{Lo}^{1}$, Kenrick Kuwahara ${ }^{1}$, Po-Ying $\mathrm{Li}^{1}$, Rajat Agrawal ${ }^{2}$, Mark S. Humayun ${ }^{2}$, Ellis Meng ${ }^{1}$ \\ University of Southern California \\ ${ }^{1}$ Viterbi School of Engineering and ${ }^{2}$ Department of Ophthalmology \\ 1042 Downey Way, DRB-140, Los Angeles, CA 90089-1111, USA \\ Tel: +1-213-821-3949, Fax: +1-213-821-3897, E-mail: rlo@usc.edu
}

\begin{abstract}
This paper presents the first passive implantable microelectromechanical systems (MEMS) device for targeted intraocular delivery of therapeutic compounds. In particular, this device addresses the treatment of chronic, difficult to reach diseases that affect the retina including retinitis pigmentosa, age-related macular degeneration, diabetic retinopathy, and glaucoma. The device is composed of three structural polymethyldisiloxane (PDMS) layers that are irreversibly bonded without the use of any adhesives. These layers form an integrated drug delivery device consisting of a refillable reservoir, tube, check valve, and suture tabs. This device requires a single implantation surgery and is capable of repeated delivery of multiple drugs. Characterization of the refillable reservoir and check valve performance is presented. Preliminary surgical implantation results of a mechanical test structure are also presented.
\end{abstract}

Keywords: drug delivery, PDMS, refillable, implant, check valve

\section{INTRODUCTION}

The ability to precisely administer pharmaceutical solutions to a specified target within the body, especially the eye, is an ongoing challenge in many areas of drug delivery. Pharmacologic management of ocular disease is particularly difficult as many factors limit drug efficacy. Traditional routes of ocular administration include systemic (oral drugs), topical (eye drops), intraocular and periocular (injections and sustained-release devices); however each of these approaches are less than optimal for chronic treatment of posterior segment eye diseases. These diseases, including retinitis pigmentosa, age-related macular degeneration, diabetic retinopathy, and glaucoma, are the leading causes for irreversible blindness in the world [1].

The surface of the eye is a significant physical barrier to medications that target intraocular treatment sites. Topical eye drops must be able to permeate through the modified mucosal membrane that covers the cornea. Less that $5 \%$ of the topically delivered pharmaceuticals will reach the intraocular space [2]. As a result, topical delivery is ineffective and often unable to achieve the required therapeutic levels at the treatment site. Oral medications face similar challenges and must overcome the blood-retinal barrier. In addition, large systemic doses applied to reach therapeutic levels may result in unwanted side effects $[1,2]$.

Intraocular injections are currently the most effective method of treatment. However, chronic diseases may require 1-3 injections per week. Patient acceptance is poor and repeated injections may contribute to dose related side effects such as hemorrhage, retinal detachment, and cataract [3]. Thus, there is a need for advanced drug delivery systems that have broad drug compatibility, are minimally invasive, refillable, and provide accurate dosing.

Drug delivery systems/devices can be broadly categorized as (1) biodegradable or nonbiodegradable implants, (2) implantable pump systems, and (3) atypical implantable systems [4]. Current ocular drug delivery devices fall either in the first or last category and have had limited success; commercially available sustained-release implants require periodic replacement and have side effects similar those associated with injections. Specifications and performance of existing conventionally manufactured ocular drug delivery devices are summarized in Table $1[3,8]$.

To our knowledge, this is the first implantable intraocular MEMS drug delivery device with refill capability. The advantages of MEMS fabrication for producing miniaturized and efficient drug delivery systems have already been realized for insulin delivery and delivery of bioactive compounds to neural tissue [5-7].

Table 1. Summary of existing ocular drug delivery devices

\begin{tabular}{|l|l|l|l|}
\hline Device & Volume & Dosage Rate & Lifetime \\
\hline $\begin{array}{l}\text { Ganciclovir } \\
\text { Implant }\end{array}$ & $5 \mathrm{mg}$ & $1-2 \mu \mathrm{g} / \mathrm{hr}$ & $\begin{array}{l}104-208 \\
\text { days }\end{array}$ \\
\hline $\begin{array}{l}\text { Corticosteroid } \\
\text { Implants }\end{array}$ & $5 \mathrm{mg}$ & $0.2 \mu \mathrm{g} / \mathrm{hr}$ & 3 years \\
\hline $\begin{array}{l}\text { Cyclosporine } \\
\text { Implants }\end{array}$ & $5 \mathrm{mg}$ & $1.3 \mu \mathrm{g} /$ day & $3-5$ years \\
\hline $\begin{array}{l}\text { Live Cell } \\
\text { Implants }\end{array}$ & $\mathrm{N} / \mathrm{A}$ & $0.2-1.0 \mathrm{~g} /$ day & 7 week study \\
\hline $\begin{array}{l}\text { Osmotic Pump } \\
\text { System }\end{array}$ & $\mathrm{N} / \mathrm{A}$ & $2.5 \mu \mathrm{l} / \mathrm{hr}$ & 28 day study \\
\hline
\end{tabular}




\section{DEVICE DESIGN AND OPERATION}

Dimensions for this ocular drug delivery device are selected such that the overall device is minimally invasive and comfortable for the patient. The device utilizes a passive delivery mechanism to eliminate the need for control electronics and thus reducing the cost of the system. In order for the device to be a viable treatment method for chronic diseases, it must be refillable to allow repeated dosing for many years. Once implanted, the device must precisely and repeatedly deliver accurate doses and hold enough medication for multiple doses prior to refilling ( every 2 months). The device should be flexible and conform to the natural curvature of the eye. An ocular drug delivery device and its placement relative to the eye (subconjunctival) are illustrated in Figure 1. An illustration of the first device prototype is shown in Figure 2.

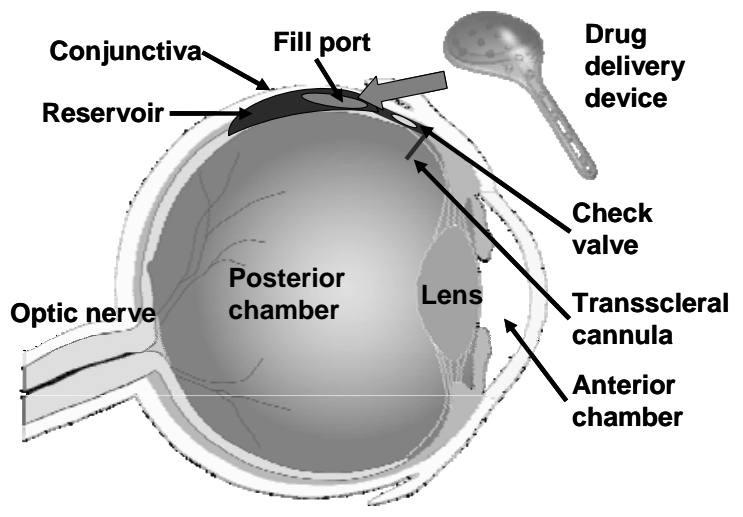

Figure 1. Concept of a refillable ocular drug delivery device

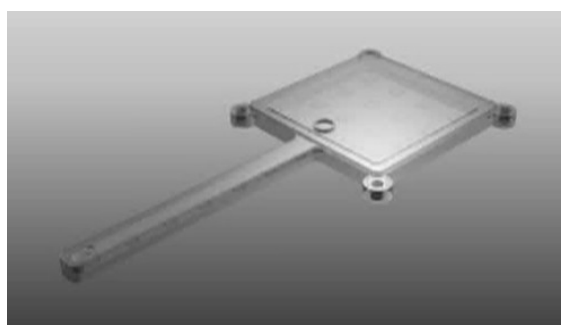

Figure 2. 3D rendered image of ocular drug delivery device

The first prototype ocular drug delivery device integrates a refillable reservoir, transscleral cannula for drug delivery, and a check valve that is located at the end of the cannula. PDMS is selected for its flexibility and ability to reseal itself after repeated punctures via a syringe needle. This material selection also enables a refillable drug reservoir. The drug delivery tube diameter is set to less than $1 \mathrm{~mm}$. For incisions of this size, the eye is able to maintain its integrity without the aid of sutures. Support posts are contained within the tube and the reservoir to prevent the top surfaces of the device from collapsing when the drug is depleted. A normally-closed check valve prevents backflow of fluids from the eye into the device. The valve opens above a certain cracking pressure allowing drug to be dosed from the reservoir to the treatment site. Suture tabs secure the device to the eye. This first prototype also serves as a surgical test model for future demonstration in rabbits.

The reservoir is secured to the top of the eye, while the shunt is inserted into either the anterior or posterior chamber (Figure 3a). A specific dose of medication is dispensed from the device when the reservoir is manually depressed by the patient's finger (Figure 3b). The reservoir can be refilled with the same or different medication without additional surgery (Figure 3c). (a)

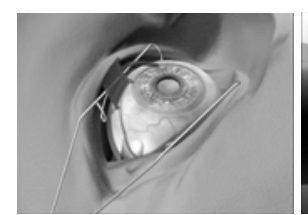

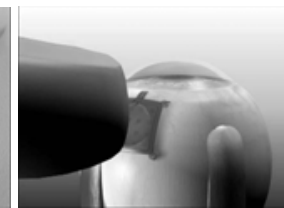

(b)

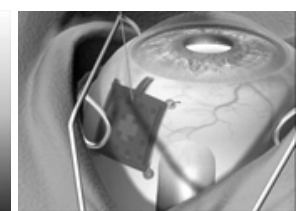

(c)
Figure 3. Illustration of device operation

\section{DEVICE FABRICATION}

The intraocular drug delivery device is composed of three individual structural layers of PDMS. The top layer defines the chamber for the refillable drug reservoir. The middle layer defines the delivery tube and check valve orifice. The bottom layer forms the base of the device outlining the refillable chamber, delivery tube, suture tabs, and check valve seat. This layer contains posts that serve as (1) mechanical supports to prevent the tube or reservoir from collapsing and (2) the valve seat for the check valve.

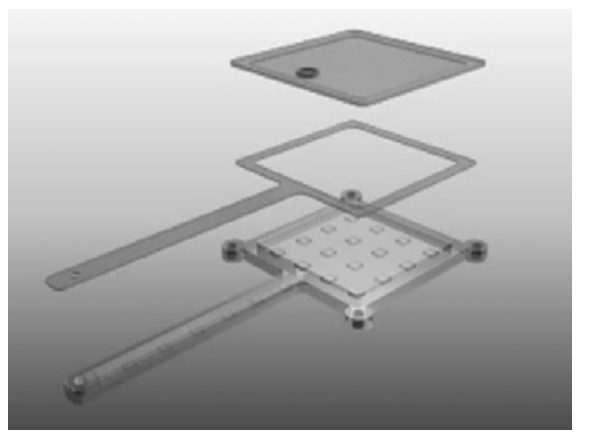

Figure 4. Exploded view of device structural layers

The top layer was fabricated using a conventionally machined mold, whereas the middle and top layers are molded from silicon masters that were etched using DRIE. The silicon masters were then coated in parylene which serves as a release layer between the PDMS and silicon. Individual layers were then bonded together assisted by either oxygen plasma [9] or wet chemical surface treatment. Both methods alter the surface chemistry of PDMS from hydrophobic to hydrophilic; this change facilitates irreversible bonding of PDMS-to-PDMS without adhesives. Figure 5 shows a completely assembled drug delivery device. 


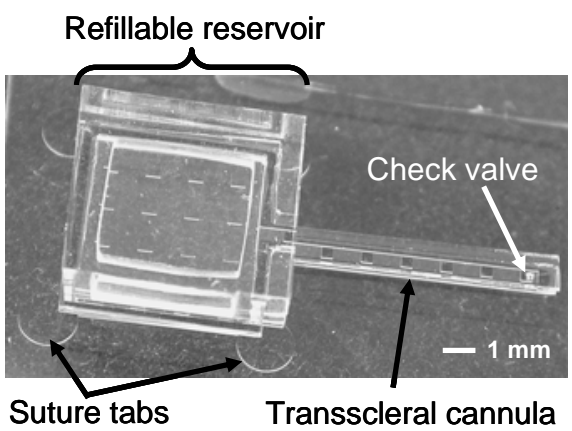

Figure 5. Assembled PDMS ocular drug delivery device

\section{RESULTS AND DISCUSSION}

\subsection{Refillable Reservoir}

Currently, the entire top layer of the reservoir serves as a refill port. Optical images of cross-sections of a single needle insertion point through silicone septa using noncoring (20 and 30 gauge) and coring (30 gauge) needles were compared (Figure 6). It is clear that a non-coring needle is able to take advantage of the self-sealing nature of the PDMS and does not remove material when puncturing PDMS.

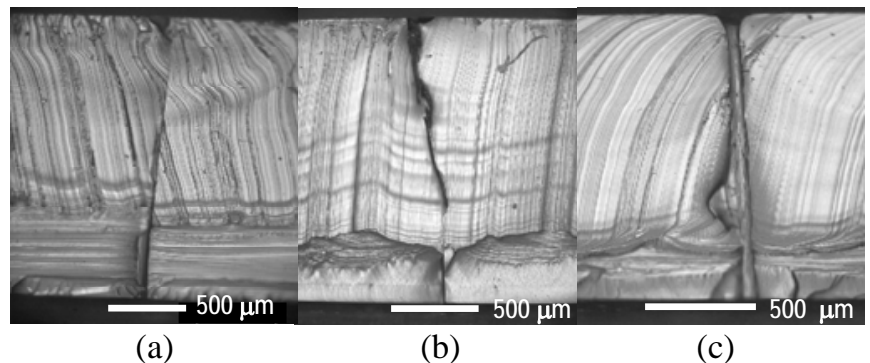

Figure 6. Optical images of cross sections at puncture sites for non-coring (a) 20 gauge and (b) 30 gauge needles and coring (c) 30 gauge needle

\subsection{Check Valve}

Dyed fluid was injected into the reservoir to verify that the assembled layers were reliably sealed. Pressure was applied to the reservoir and fluid flow was optically monitored as it moved down the tube and out of the check valve (Figure 7).

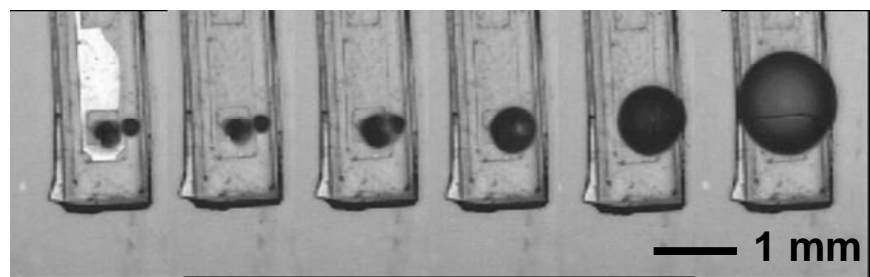

Figure 7. Time lapse images of check valve operation

The operating characteristics of the check valve were determined by clamping the device in a custom testing apparatus and attaching the fixture to a water chamber pressurized by a nitrogen cylinder (Figure 8 ). In such a manner, pressurized water is forced through the delivery tube and out of the check valve. Flow rate is measured by timing fluid passage through a precision calibrated pipette. The valve's cracking pressure (62 kPa) and flow rate vs. applied pressure for DI water flow are presented in Figure 9. The check valve requires optimization to obtain operation at physiologically-compatible cracking pressures.

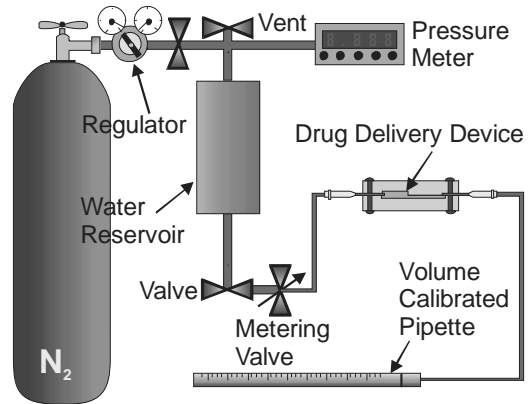

(a)

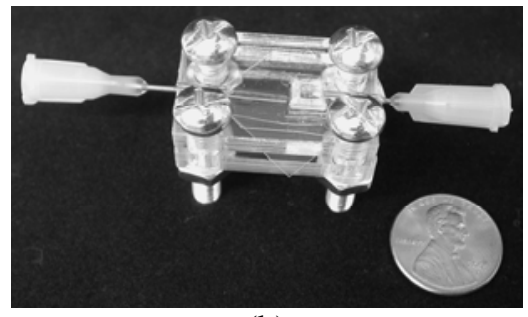

(b)

Figure 8. (a) Experimental apparatus for determining check valve performance and (b) fixture for holding the device

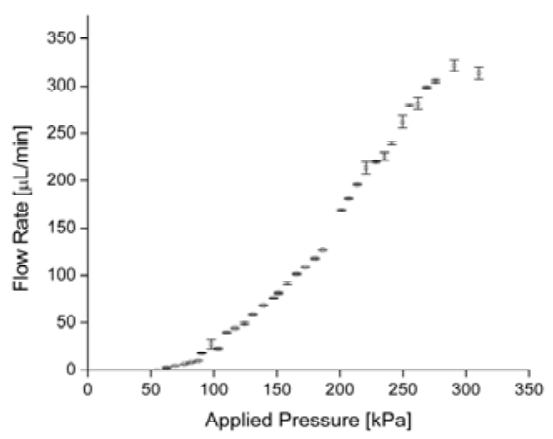

Figure 9. DI water flow rate vs. applied pressure for the integrated check valve (Mean \pm SE, $n=4$ )

\subsection{Surgical Implantation}

The prototype device was utilized as a surgical test model for mechanical characterization from both surgical handling and operation perspectives. The device was implanted into an enucleated porcine eye. First, the reservoir portion was secured to the eye wall using the suture tabs and then the tube was directed into the anterior chamber through a small incision at the limbus (Figure 10). Based on preliminary surgical results, the device geometry requires adjustment to facilitate the implantation surgery. Various device geometries are being considered and will be evaluated in 
enucleated porcine eyes. In vivo experiments in rabbits are planned to demonstrate drug delivery.

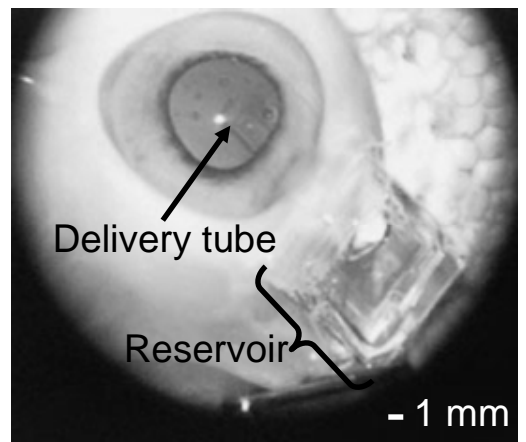

Figure 10. Drug delivery device implanted in enucleated porcine eye

\section{FUTURE WORK}

The concept for the second generation device is shown in Figure 11 and will conform to the eye contours and integrate flow regulating components that will deliver specific and repeatable dose volumes. It will also incorporate geometrical changes suggested by ophthalmic surgeons to facilitate implantation. The edges of device will be rounded in order to distribute stress and minimize dead volume. The refill port will be relocated to maximize accessibility after the device is implanted. Other materials for the refill port and device body are being investigated to further extend lifetime and mechanical robustness under surgical handling. A surgical procedure utilizing phenylephrine, a quick-acting dilating agent, to validate device functionality in vivo will be developed. Future versions will also explore electronically controlled pumping to achieve drug delivery.

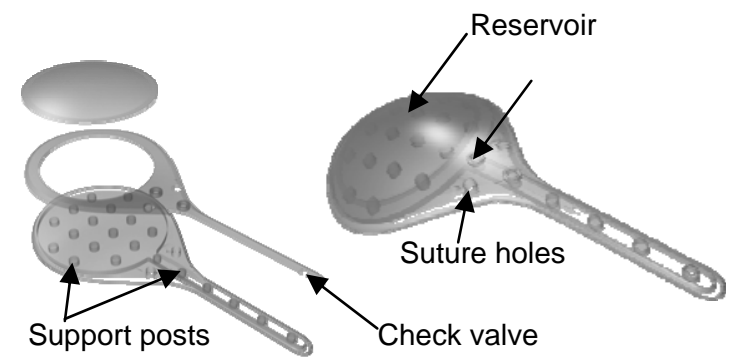

Figure 11. Exploded and assembled views of second generation device.

\section{CONCLUSION}

This device is the first reported passive MEMS refillable intraocular drug delivery device. Its miniature size and refill feature promises more effective drug treatment for chronic eye diseases. This multi-layer PDMS device requires only a single implantation surgery and can be repeatedly refilled with the same or different medications. Characterization of the device and preliminary surgical implantation results of the mechanical model are presented. Future work includes in vivo demonstration of ocular drug delivery and application of this drug delivery concept to treat conditions in other physiological systems.

\section{ACKNOWLEDGEMENT}

This work was supported in part by the Engineering Research Centers Program of the NSF under Award Number EEC-0310723 and Bausch \& Lomb. The authors would like to thank Tuan Hoang, Mei Nickles, Tun Min Soe, Lilian Tran, and Joy Wong for their contributions to this work.

\section{REFERENCES}

[1] D. H. Geroski and H. F. Edelhauser, "Drug delivery for posterior segment eye disease," Invest Ophthalmol Vis Sci, vol. 41, pp. 961-4, 2000.

[2] D. C. Metrikin and R. Anand, "Intravitreal drug administration with depot devices," Curr Opin Ophthalmol, vol. 5, pp. 21-9, 1994.

[3] S. Lee, P. Yuan, and M. R. Robinson, "Ocular Implants for Drug Delivery," Encyclopedia of Biomaterials and Biomedical Engineering, pp. 1105-1119, 2004.

[4] A. K. Dash and G. C. Cudworth, 2nd, "Therapeutic applications of implantable drug delivery systems," $J$ Pharmacol Toxicol Methods, vol. 40, pp. 1-12, 1998.

[5] A. C. R. Grayson, R. S. Shawgo, Y. W. Li, and M. J. Cima, "Electronic MEMS for triggered delivery," Advanced Drug Delivery Reviews, vol. 56, pp. 173-184, 2004.

[6] S. Z. Razzacki, P. K. Thwar, M. Yang, V. M. Ugaz, and M. A. Burns, "Integrated microsystems for controlled drug delivery," Advanced Drug Delivery Reviews, vol. 56, pp. 185-198, 2004.

[7] B. Ziaie, A. Baldi, M. Lei, Y. D. Gu, and R. A. Siegel, "Hard and soft micromachining for BioMEMS: review of techniques and examples of applications in microfluidics and drug delivery," Advanced Drug Delivery Reviews, vol. 56, pp. 145-172, 2004.

[8] J. Ambati, E. S. Gragoudas, J. W. Miller, T. T. You, K. Miyamoto, F. C. Delori, and A. P. Adamis, "Transscleral delivery of bioactive protein to the choroid and retina," Investigative Ophthalmology \& Visual Science, vol. 41, pp. 1186-1191, 2000.

[9] D. C. Duffy, J. C. McDonald, O. J. A. Schueller, and G. M. Whitesides, "Rapid prototyping of microfluidic systems in poly(dimethylsiloxane)," Analytical Chemistry, vol. 70, pp. 4974-4984, 1998. 